\title{
Application of Analytical Geometry in Big data of Tehran Municipality's Waste Management AVL System
}

\author{
Davarnia, Sepehr; Haji, Alireza; MortezaGhasemi, Atrin \\ IAU TNB, Sharif University of Technology, Sharif University of Technology
}

\begin{abstract}
Nowadays the world is experiencing a fast growing of urbanization and population in cities. One of the biggest challenges caused by this rapid growth is protecting the environment. To overcome and solve this issue, researchers are trying various methods. One of these methods is using ICT in city management, which leads to a smart city concept. In a highly populated city, it is required to utilize efficient waste management methods in order to gather, transport and process the waste produced. Due to the high rate of waste generation in big cities, optimal logistic plans to reduce traffic caused by waste gathering vehicles are required. To build these logistic plans historical data of as-is conditions is necessary which can be gathered using GPS technology to record the location and speed of each vehicle using a desired time frequency (AVL systems). The purpose of this research is to identify inefficiencies of waste collection routes in Tehran and to provide recommendations in order to reduce these inefficiencies. This system has been implemented in Tehran for 5 years using 3000 vehicles. Working on almost 1,000,000,000 data samples, some fundamental problems in the waste management of the city are discovered. It is concluded that the route plan of the transportation vehicles was not designed properly and the necessary monitoring was not performed. Some waste gathering vehicles did not even follow the designated path. The resulting data driven policy helps to reduce these inefficiencies of waste management by improving routes taken by waste gathering vehicles resulting in less fuel consumption and less traffic.
\end{abstract}

Keywords: data; waste management; smart city; waste gathering vehicles; logistic 